\title{
IoT-Based Reusable Medical Suit for Daily Life Use in the Era of COVID-19
}

\author{
Abdelhamied A. Ateya ${ }^{1,2}$, Abeer D. Algarni ${ }^{1}$, Hanaa A. Abdallah ${ }^{1,2}$ and Naglaa F. Soliman ${ }^{1,2, *}$ \\ ${ }^{1}$ Department of Information Technology, College of Computer and Information Sciences, Princess Nourah Bint Abdulrahman \\ University, Riyadh, 84428, Saudi Arabia \\ ${ }^{2}$ Department of Electronics and Communications Engineering, Zagazig University, Zagazig, 44519, Egypt \\ *Corresponding Author: Naglaa F. Soliman. Email: nfsoliman@pnu.edu.sa \\ Received: 30 June 2021; Accepted: 16 August 2021
}

\begin{abstract}
Coronavirus disease (COVID-19) is a big problem that scares people all over the world. Life over the world has changed, new aspects for daily life have been introduced. A main problem with COVID-19 is the way it spreads. Covid-19 spreads, primarily, through contact with an infected person when they cough or sneeze, or with an infected surface. Thus, a novel way to make a protection against COVID-19 is to stay away or make yourself isolated from infected people and surfaces. To this end, this work, mainly, aims to design and develop a novel auto-sterilized suit embedded with some medical sensors and other Internet of Things (IoT) devices to provide the required level of isolation, safety, tracking and monitoring of COVID-19 and other pandemic diseases. The developed suit is an auto-sterilized suit for medical purposes and for daily life use. The sterilizing process of the suit is controlled by the IoT paradigm to provide the required control and interface in an automated way. According to the location of the user, wearing the suit, an appropriate sterilizing mode is activated automatically and the suit is sterilized via distributed nozzles over the suit. Furthermore, the distributed medical sensors represent a wireless body area network (WBAN) that is integrated with an IoT gateway to provide periodic measures of medical healthcare parameters such as body temperature, breathing rate, oxygen saturation level and pulse rate. These measures are used to identify the user's health and the probability of being infected by COVID-19. All measures are transferred to the remote IoT cloud to analyze these data and monitor people around the day. In case of unusual measures, users are moved among three databases associated with health, infected and properly infected users. The suit is under prototyping and the work is mainly introduced to present the design stages.
\end{abstract}

Keywords: COVID-19; IoT; Auto-sterilizing; WBAN; remote healthcare; health monitoring

\section{Introduction}

According to the world health report 65, the coronavirus (COVID-19) spreads to 202 countries with more than half million confirmed cases and near to twenty thousand deaths; this is by 25 of March

This work is licensed under a Creative Commons Attribution 4.0 International License, which permits unrestricted use, distribution, and reproduction in any medium, provided the original work is properly cited. 
2020 [1]. This made the World Health Organization (WHO) declare COVID-19 as a global pandemic; turning from global public health emergency announced by the end of January 2020. The number of confirmed cases increases exponentially in a dramatic way, which changes the world and all daily life activates everywhere [2].

COVID-19 is an unanticipated disaster that pushes all governments, companies, and academics to search and develop new ways and drugs for monitoring, controlling and treating infected people. All around the world work to find the cure, and all expectations lead to the worst scenario of waiting for months until the cure be ready [3]. To this time, and even when the cure is ready, it is important to monitor infected and non-infected people. Furthermore, saving non-infected people, decreasing number of infected cases, and controlling the pandemic are high demands. The early symptoms of COVID-19 include recording high body temperature, changing the breath rate and pulse rate beside tiredness, and dry cough [4].

The recent advances in biomedical sensory manufacturing led to a big revolution in remote healthcare systems. Wireless body area network (WBAN) represents the communication paradigm that covers the interaction and communications of biomedical sensors over the appropriate wireless channel [5]. Introducing Internet of Things (IoT) and WBANs for healthcare provides high-efficient solution, and increases the efficiency of the national health system [6].

The IoT transformation is remodeling modern healthcare systems by joining technological, economic, and social prospects. Healthcare services are changing from traditional to more personalized healthcare systems, making it easier to diagnose, treat, and monitor patients [7]. The current global pandemic threat, caused by the emerging serious respiratory syndrome coronavirus, poses the biggest global public health problem, since the 1918 influenza pandemic outbreak [2]. There has been a rapid effort in various research communities to leverage a wide variety of technologies to tackle this global threat, since the pandemic began. IoT technology is one of the pioneers in this field. Within the COVID-19 background, IoT-enabled applications are used in the sense of COVID-19 to minimize the potential spread of COVID19 to others, through early detection, patient monitoring, and the practice of specified protocols after patient recovery [8].

The main contribution of this work is to design and develop two main systems, based on IoT, to work against the spread of COVID-19. The first system is an auto-sterilizing suit that can be used by anyone to avoid the infection of COVID-19. This turns the market available disposable medical suits into reusable, which reduces the cost and control the infection. As there is no need to find a place for throwing the used suit, which may be infected by COVID-19. An auto-sterilized system will periodically sterilize the suite in a cost-efficient way. The second system is a remote, reliable healthcare system based on the IoT paradigm. The healthcare system consists of distributed wireless biomedical sensors connected to the suit, to measure and live monitoring medical parameters. The considered sensors are mainly used to measure body temperature, breath rate and pulse rate. These measures are efficient to make a primary diagnosis of COVID-19. We can summarize the contributions of the work in the following points.

1. Building a WBAN integrated with IoT.

2. Developing an auto-sterilizing system.

3. Designing and integrating location module.

4. Developing the IoT gateway.

5. Designing and developing the IoT-cloud, i.e., Application server, with an operator interface to monitor the collected data and process it.

6. System level integration.

7. Performance assessment. 


\section{Background and Related Works}

WBAN is the recent paradigm that introduces improved methods for diagnosis of different dangerous diseases, and monitoring human's health. Using WBAN, user's healthcare parameters can be monitored in real-time, and any health problem can be detected concurrently. WBAN is an emerging technology; thus, until now, different problems need to be tackled. The main enabler of remote health monitoring is the WBAN [9], which incorporates small sensors and wireless communication technology. Wearable and implantable devices are used to gather physiological data, in order to constantly monitor the physical conditions of people [10].

The planet has been dealing with the pandemic, since the beginning of 2020, by seeking to monitor the unprecedented spread of the virus, and creating a vaccine. Because most of the attempts to find a cure or regulate the spread of COVID-19 have not shown satisfactory results to date, there is a strong demand for global monitoring of symptomatic patients. IoT technology has gained considerable attention in recent years in the healthcare sector, where it plays an important role in the various stages of different infectious diseases [7]. In the current pandemic, as the COVID-19 contingency is strong, there is an urgent need for patients to be proactively linked to their physicians [2].

Remote healthcare systems gain high attention, with the recent advances of sensory manufacturing, and the great evolution in telecommunication industry. There are many literatures that consider developing remote healthcare systems based on WBAN [11-13]. However, rare literatures consider integrating WBAN with IoT paradigm. In this section, we introduce the recent related works consider developing an IoT based system for monitoring and tracking COVID-19. Furthermore, recent literatures that consider developing remote healthcare systems based on IoT are introduced.

In [14], Jung et al. developed a system for investigating and tracking COVID-19, using virtual IoT nodes installed on the user's smartphone. The system deploys a software defined networking (SDN) platform based on a controller-centric scheme. The system mainly considers COVID-19 tracking; however, our developed framework considers developing a remote healthcare system, besides tracking and monitoring COVID-19. The system depends on virtual IoT nodes built over smartphones, not a real IoT system.

In [15], Siriwardhana et al. developed a framework for integrating IoT with 5G, to effectively combat the spreading of Covid-19 pandemic. Moreover, authors presented numerous use cases, and achieved solutions to different problems, in many fields, results due to pandemic such as healthcare, self-isolation, education problem, and government services. The novelty of our proposed work, compared to this work, comes from the consideration of WBAN and auto-sterilizing process.

In [16], Ndiaye et al. presented an overview research up on the development of the E-health system that depends on the sensors and the impact of COVID-19 on the IoT market in the future. Likewise, IoT improves the availability of eminence precise data in such a way that there is a bad need of security and robustness of data in such systems. The work mainly introduces a general point of view of how IoT can be used to monitor COVID-19, and the reverse direction of how COVID-19 affects the IoT market and solutions in the future. This is completely differing from our proposed framework, since we provide a real system with an experimental evaluation and furthermore, we introduce an auto-sterilizing system for the first time.

In [17] Priambodo et al. conducted a real-time IoT system for health monitoring and data surveillance. In this system, home pulse oximeter is acquired the $\mathrm{SpO} 2$ and heart rate measurement values, and send this data through an IoT gateway using android application. Then, it adds the identity and location of the patient to the obtained data. Finally, the data is transmitted to the server through a Bluetooth Low Power (BLP) device. 
The novelty of our proposed work comes from integrating the WBAN with the IoT to provide reliable, and cost-efficient remote healthcare system. Furthermore, to the best of our knowledge, the proposed work is the first one that aims to turn the disposable medical suit to reusable one, with an auto-sterilized system and thus, achieving two main goals; cost efficiency and infection control.

\section{Proposed IoT-Based Suit}

In this section, the developed IoT-based suit, with the integrated WBAN and auto-sterilizing system, is introduced. The developed IoT-based suit can be viewed as three integrated parts; data acquisition part, autosterilizing part, and controlling and management part. The three parts are integrated with the general structure of the IoT network, since the control and management unit is embedded with the IoT gateway. Fig. 1 presents the end-to-end system structure of the developed IoT-based suit. Furthermore, the three common layers of IoT networks are introduced; perception layer, network layer, and application layer.

(a)
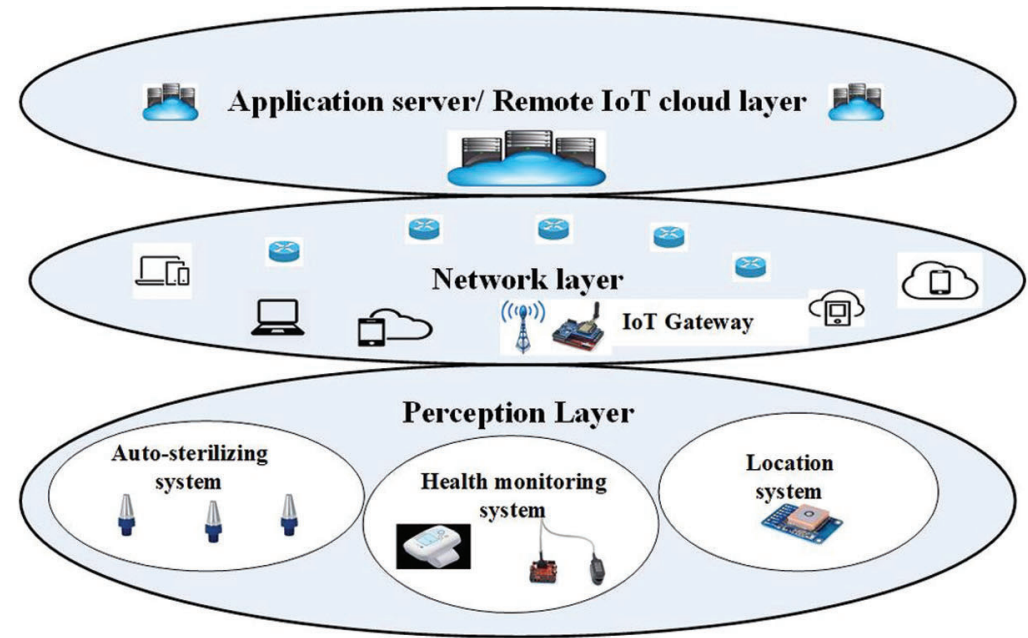

(b)

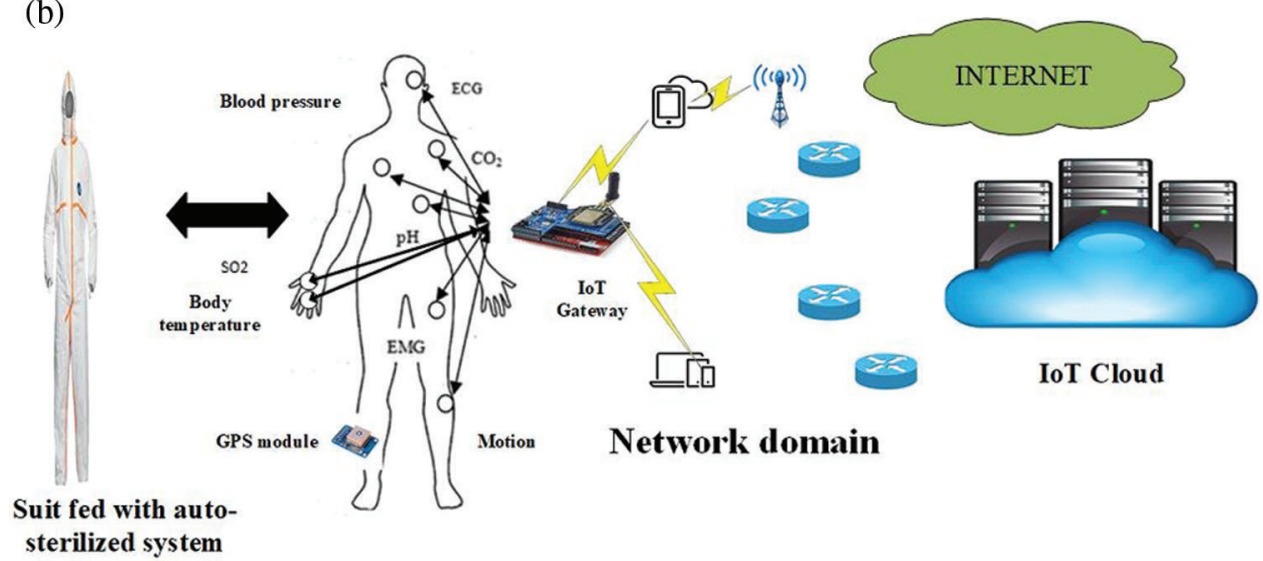

Figure 1: End-to-end system structure of the proposed IoT-based suit: (a) Main layers of the proposed IoT system; (b) Main components of the proposed IoT-based suit

\subsection{Data Acquisition Part}

This part represents the perception layer of the developed IoT system that contains the end IoT devices, e.g., biomedical sensors. Data acquisition part represents the healthcare monitoring layer that contains all 
connected biomedical sensors deployed for measuring healthcare parameters and identifying COVID-19. Biomedical sensors, deployed in this subsystem, are either primary or secondary. Primary biomedical sensors include body temperature sensor, air flow rate sensor, SO2 sensor, and pulse rate sensors. These sensors are important for monitoring health status and COVID-19, since these sensors provide measures for human temperature, breathing rate, level of oxygen saturation in blood, and pulse rate. These measures are used to identify the probability of being infected with COVID-19.

The other category of sensors, deployed in the perception layer of the proposed system, is secondary optional sensors. These sensors may be deployed for suits, with higher costs, to measure biomedical variables and provide higher medical care. Optional sensors include Electrocardiogram (ECG) sensors, Electroencephalogram (EEG) sensors, Mussel sensors and Glucometer sensors. With the recent advances in sensory manufacturing and biomedical sensors, there are enormous number of available market biomedical sensors that support either wireless or wired communication interface or both of them. Moreover, these biomedical sensors become of small sizes, which facilitates the implementation and integration.

Besides biomedical sensors, the developed IoT-based medical suit deploys additional hardware responsible for estimating the user's location at any time, which is referred to as the location system. A small cost-efficient and low energy GPS module can be deployed as the location system.

\subsection{Auto-Sterilizing Part}

The second main part of the proposed IoT-based suit is the auto-sterilizing system, which is responsible for periodically sterilizing the suit, without human intervention. This is why we call it auto-sterilizing, since it proceeds the sterilizing process automatically based on a predefined algorithm, explained later in this section, implemented by the control unit of the suit. The auto-sterilizing system is deployed to achieve the following functions.

1. Auto-sterilizing of the suit by using alcohol. Each point in the suit should be sterilized by alcohol, periodically, through distributed controlled nozzles.

2. Controlling the time of re-sterilizing and the amount of passed alcohol for the sterilization, based on the location of the user and the surrounded environment.

3. The process of sterilizing is done periodically, with heterogeneous time intervals selected and estimated by a controller.

4. The whole process is cost-efficient and health-efficient. This can be done by controlling the amount of passed alcohol to nozzles.

The general structure of the auto-sterilizing system is introduced in Fig. 2. As presented in the figure, the system deploys distributed nozzles over the suit, in a predefined geometry to cover the whole suit. All nozzles are connected to a thin-flat alcohol tank via a hidden thin pipeline system. The pipeline system is connected to the alcohol tank and the pump through a controller to control the amount of passed alcohol, the nozzle orientation, and the time of operation. All the main components of the sterilizing system are chosen in such a way that it can be implemented in the inner coating of the suit. The standard announced sizes of medical coats and protective cloths are introduced in [18,19], as per the World Health Organization. The proposed medical suit can be, geometrically, viewed as six main sections; Face, Neck, Sleeve, Chest, Hips, and, Legs. The distribution of alcohol nozzles is a function of suit geometry and its size, in a way that achieves the required alcohol spreading process. The main objective of the auto-sterilizing system is to automatically sterilizing the suit, in a periodic form and based on the person's location, by spreading alcohol everywhere in the suit; in a cost-efficient way, without wasting resources. We made a survey of current market available nozzles that can be used for alcohol distribution. The required nozzles should be of small size to facilitate the integration with the pipeline system and the control unit. 


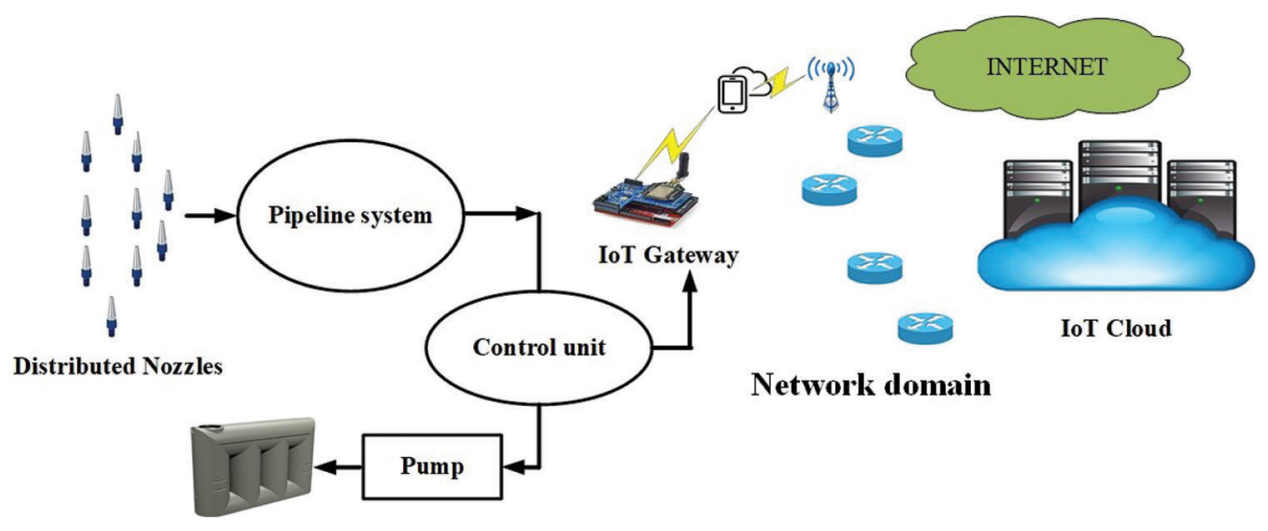

Figure 2: General structure of the auto-sterilizing system

Among all available spray nozzles for alcohol, the full-cone type represents the most appropriate one for the proposed medical IoT-based suit. This is because of its size, and ease of integration and implementation with the developed system. The mechanism of operation of full cone spray nozzles is to distribute the droplets into a volume that is figured by a cone with a center at the orifice of the nozzle. This full cone spray pattern represents an efficient distribution pattern for many industrial processes that require liquid spraying. Since this type of liquid spray nozzles is commonly used, it evolves rapidly and many kinds of full-cone nozzles become market available.

The available market full cone spray nozzles can be categorized into three main categories; standard full cone, spiral full cone, and multiple full cone [20]. The standard full cone spray nozzle employs a vane, with predefined shape at the inlet of the nozzle, this is to achieve a rotational speed for the flow of fluid passes through the nozzle. This produces force and torque that sprays the alcohol through the orifice of the nozzle to a cone-shaped area with a certain angle. Practical market available standard full cone spray nozzles achieve spray cones with angles vary from 15 to 120 degree. The second type of full cone spray nozzles is the spiral full cone nozzles that achieves a spray of spiral shape in a conical volume. The main disadvantage with this type is the nonuniform distribution. The third type of full cone nozzles is the multiple full cone spray nozzles, which is multiples of standard full cone. This type is used to achieve a large spray angle that cannot be achieved by the standard full cone. This can be achieved by deploying several nozzles oriented in different directions and grouped in a cluster. However, this type deploys several nozzles in a cluster, its size is small compared to other two types of full cone spray nozzles. Multiple full cone nozzles can achieve a spray angle of 360 degrees, with only seven connected nozzles in one cluster.

For the proposed medical suit, the most efficient and appropriate spray nozzle is the multiple full cone spray nozzle introduced in [21]. The selected nozzle deploys one cluster with seven nozzles per cluster. We can summarize the reasons of why we select this type of nozzles to the proposed auto sterilizing system, in the following points.

1. It can achieve a cone angle of 360-degree, which means that it can spread the alcohol over the 360-degree surrounded area.

2. It is a micro nozzle with a very small-scale dimension, in millimeter range, which facilitates the integration and implementation of such nozzles with the medical suit.

3. These nozzles are cost-efficient, which makes the overall cost of the suit efficient.

These 360-degree spray nozzles are distributed over the six main parts of the proposed medical suit, in a way that achieves a full alcohol distribution. The position and the number of deployed nozzles is a function of 
suit dimensions. According to the available market sizes of medical suits and the announced WHO specifications of protective wear, we select the medium size, for prototyping our proposed protective reusable suit $[18,19]$. Fig. 3 introduces the distribution of the multiple full cone spray nozzles over the six main parts of the suit in a way that achieves the full alcohol distribution, for the medium size of protective clothes dimensions indicated in [19]. Moreover, two nozzles for hand sterilizing are embedded with the end of each sleeve. One of them for sterilizing the upper-surface of the hand and the other is for the lower-surface of the hand; this is carried out either with gloves or without. However, deploying a protective hand wear, e.g., medical gloves, requires fewer sterilizing times than normal hands without no protective gloves. The controller manages the sterilizing process according to the process indicated later in this section.

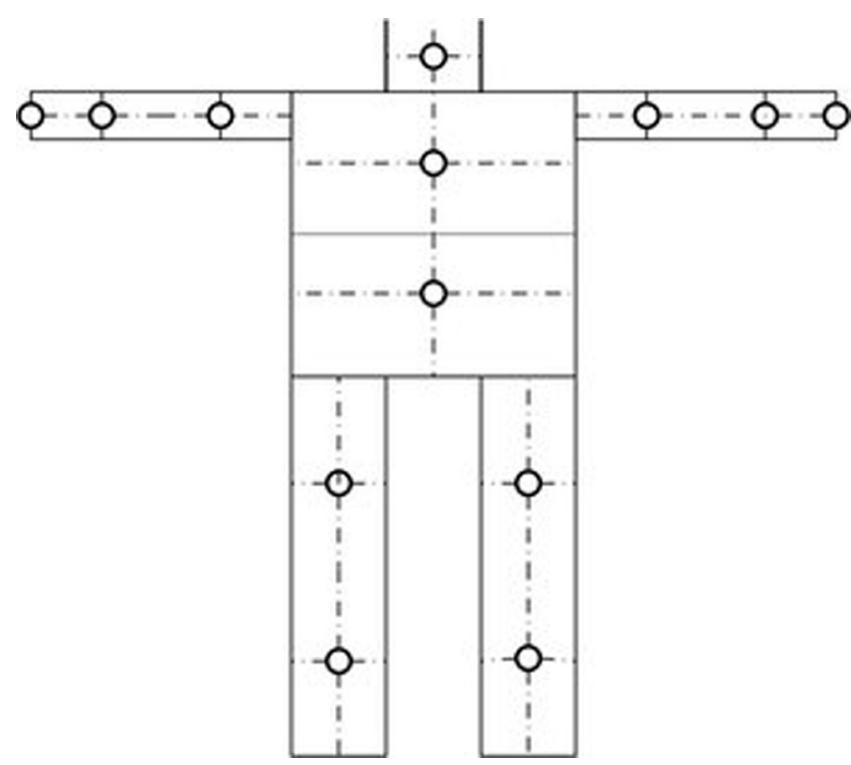

Figure 3: Layout of the distribution of micro-full cone nozzles over the suit

Distributed located nozzles are connected to the alcohol tank via a thin pipeline system. The pipeline system can be viewed as two main parts; pipeline system for hand sterilizing and pipeline system for medical suit sterilizing. Each pipeline part is connected to the alcohol tank, via a pump, that has an interface to the controller. The last part of the sterilizing system is the alcohol tank, which is a small size flat tank of $500 \mathrm{ml}$ volume. The tank is thin-flat so as to facilitate the implementation and integration with other parts, and with the suit. It is embedded in the back of the medical suit, and coated with the suit material to be seamless for the end-user. Furthermore, the tank has an orifice that represents the user interface for recharging the alcohol.

\subsection{Control and Management Part}

The third part of the developed medical IoT-based suit is the control and management system. This part is responsible for controlling the overall system, and managing the collected data from the perception layer. We can introduce all functions achieved by the control and management system in the following points.

1. Controlling the operation of the auto-sterilizing system, based on predefined required specifications, which is implemented in algorithm 1 ,

2. Data gathering and processing of the recorded measures collected by the distributed biomedical sensors deployed in the perception layer, 
3. Data offloading to the application server, i.e., IoT-Cloud, and

4. Ease of implementation and integration.

A small-scale, cost-efficient IoT gateway can implement all functions required by the control and management system. The considered IoT gateway supports an appropriate communication interface as per deployed sensors, e.g., LoRa or NB-IoT, for collecting data from the distributed medical sensor and the GPS module. Gateways that support multiple communication interfaces can be used to enable the deployment of biomedical sensors, with heterogeneous communication interfaces; however, this comes on the overall system cost. These data are preprocessed, and then forwarded to the application server via the Internet. The IoT gateway has an interface to the Internet, through cellular network.

Besides data gathering and processing, IoT gateway represents the control unit of the developed medical suit that is responsible for controlling auto-sterilizing process. The gateway implements algorithm 1, which is responsible for controlling the auto-sterilizing process. As introduced in algorithm 1, the gateway implements three modes of sterilizing, based on the user's location and the surrounding field. The first mode is the usual sterilizing mode, at which, the sterilizing process is held every predefined interval, $\mathrm{T}_{\mathrm{U}}$.

The second mode is introduced for dense urban regions that requires sterilizing at smaller intervals, since the probability of virus and infection increases in such crowded regions. The time interval at which the auto-sterilizing process is held in the second mode is $\mathrm{T}_{\mathrm{D}}$. The third mode is introduced for ultra-dense urban regions, where density of people is very high and the probability of virus spreading and infection is very high. This mode requires sterilizing with higher frequency; thus, the auto-sterilizing process is carried out over a short interval defined as $\mathrm{T}_{\mathrm{UD}}$. The three times, i.e., $\mathrm{T}_{\mathrm{U}}, \mathrm{T}_{\mathrm{D}}, \mathrm{T}_{\mathrm{UD}}$, are predefined and can be reconfigured via the application server. Once an update of these times is performed, the application server sends the gateway that modifies the updated parameter. Each mode has an associated database stored in the application server that contains locations correspond to each mode. The controller selects the sterilizing mode, by comparing the current location recorded by the GPS module with the locations mentioned in the database. It should be mentioned that the three introduced sterilizing modes can be extended for large cities, more modes can be added and defined, in the same way as per the introduced algorithm.

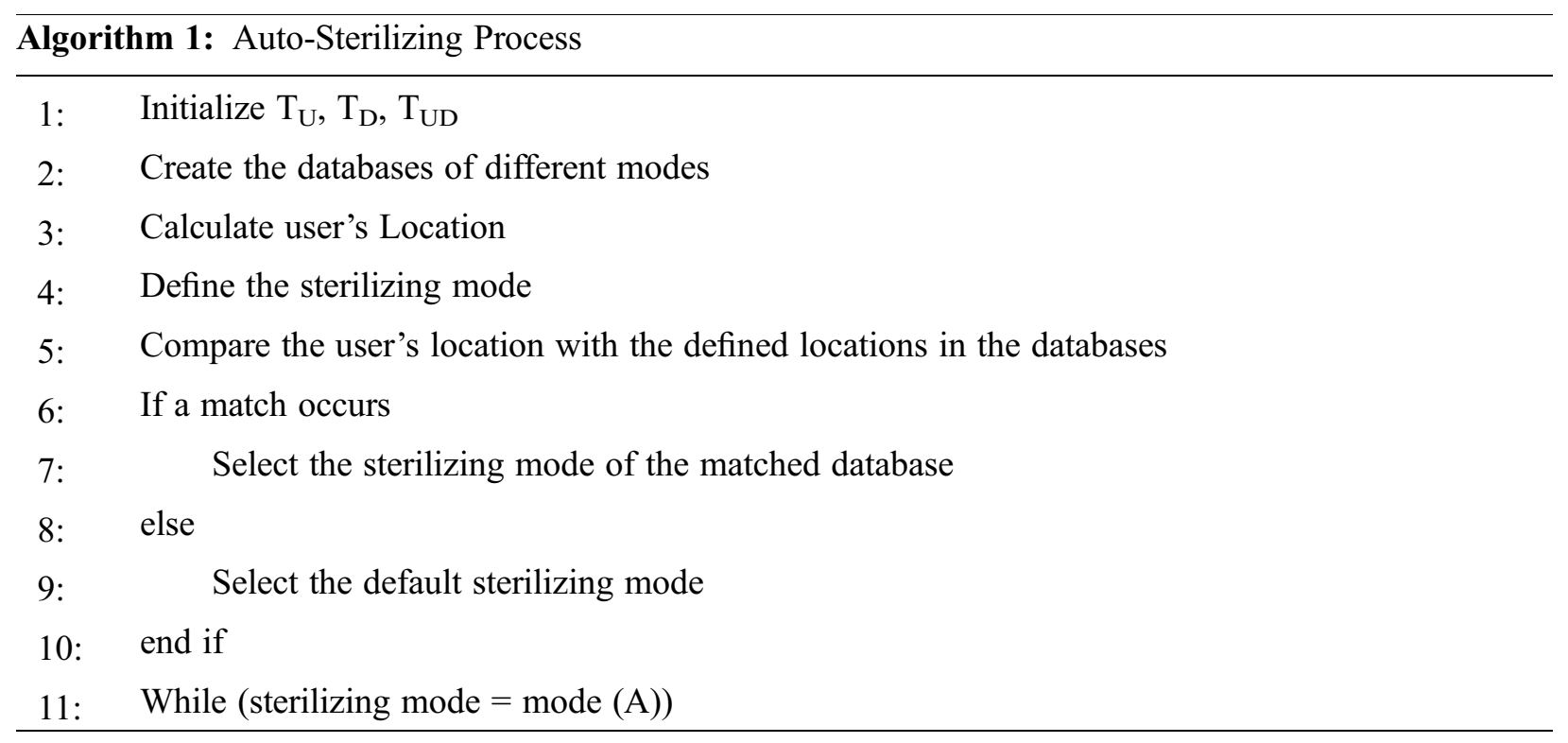




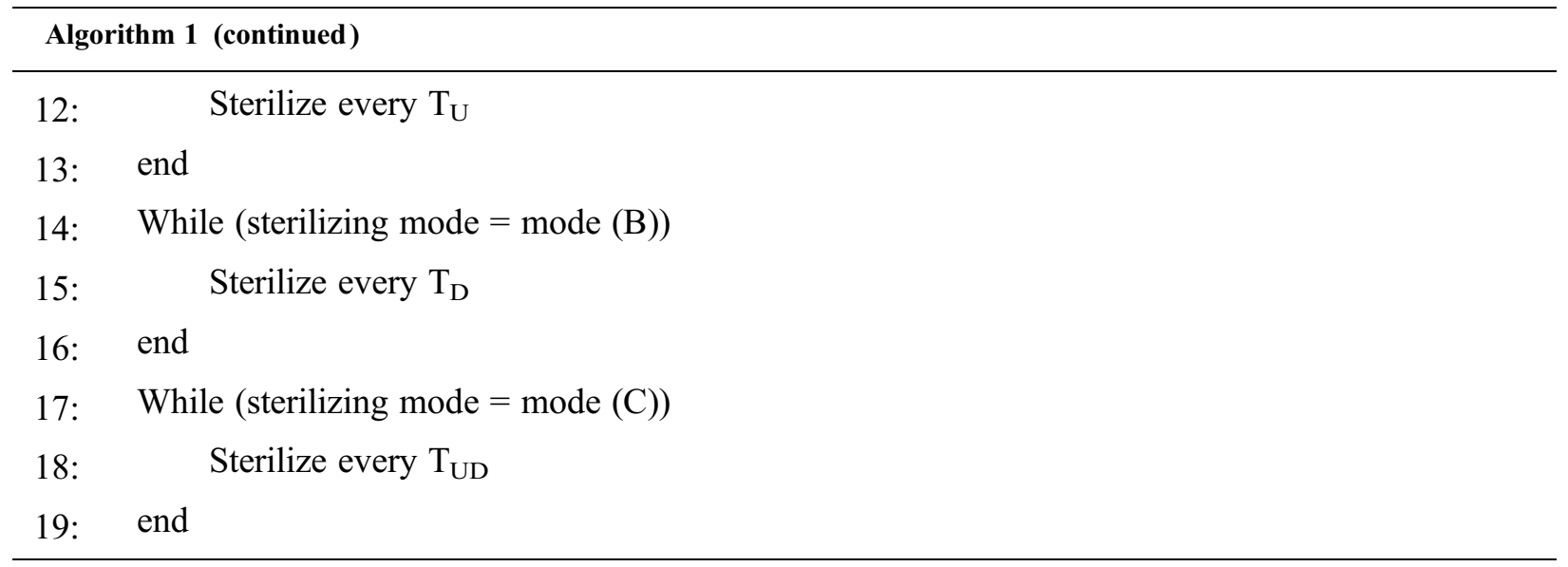

\subsection{Application Server}

This part represents the application layer that contains the IoT cloud. A remote healthcare sever can be used as the application server with two authorized logins; one by the user and the other is by the authorized healthcare institutes. The application server categorizes all registered users into three main categories, based on the health status and the medical history. The first category is represented by the database that contains all healthy users with normal measures. The second category contains the probably infected users; while the third category is introduced for confirmed infected cases. All users are categorized based on their measures and records, and once the measures change, over predefined threshold levels, the user moves to the second database. In this case, a notification is sent to the user, after repeated measures, and as well to a governmental institution responsible for this purpose.

\section{Performance Evaluation}

For performance evaluation of the developed IoT-based suit, the system is experimentally evaluated for two main scenarios. The first scenario is introduced for evaluating the performance of the wireless body area network embedded with the suit. This includes the IoT network with all medical sensors. The second scenario is introduced for evaluating the performance of the auto-sterilizing system.

This section is divided into two sub-sections; the first is for the experimental setup and the evaluation metrics, while the second sub-section is for presenting the experimental results.

\subsection{Experimental Setup}

For performance evaluation of the IoT-based WBAN connected to the medical suit, we built ten testbeds of heterogeneous medical sensors and IoT gateways. Each testbed represents a WBAN that is connected to a suit. Each suit, i.e., testbed, has its IoT gateway that collects the medical data from the distributed medical sensors and passes it to the application server. Tab. 1 introduces the considered sensors and IoT gateway for testbeds and their specifications. Each testbed was connected to a medical suit and tested for ten persons over 15 days, for an average of 8 hours per day. This is for heterogeneous activities and locations.

For performance evaluation of the auto-sterilizing system, the developed controller-nozzle system is tested for the city of Riyadh, in Saudi Arabia. The map of Riyadh city is divided into four main regions, each of which is associated with a sterilizing mode. The first type of regions is the indoor that includes all government facilities, schools, medical centers, and shopping centers. Such places are assumed to be crowded and thus, the sterilizing process should be performed with high frequency. Once the user is 
detected in such regions, the sterilizing process corresponds to indoor locations is activated. The second category represents the dense urban regions that includes crowded outdoor places. Such regions require frequent sterilizing process, due to crowd.

Table 1: Specifications of the developed testbed

\begin{tabular}{lll}
\hline Node & Vendor & Communication interface \\
\hline IoT gateway & MultiTech Conduit ${ }^{\circledR}$ & All \\
GPS module & Global-sat LT & LoRaWAN/ SF=10 \\
Pulse oximeter & Signals/ Viatomtech & Bluetooth \\
Body temperature sensor & Signals/Texas & Inter-Integrated Circuit (I2C) \\
Heart rate sensor & Signals & I2C \\
Galvanic skin response / GSR & Signals /Shimmer & I2C \\
Muscle sensor & Signals/Neural bioelectric & Bluetooth/ I2C \\
EEG & Signals/ Muse & Bluetooth/ I2C \\
ECG-portable & Signals/FaceLake & Bluetooth/ I2C \\
Application server / IoT cloud & Fujitsu & - \\
\hline
\end{tabular}

The third category is the urban regions that include regions with medium density. These regions include medium density regions such as uncrowded streets and parks. The sterilizing mode, corresponding to urban regions, sprays alcohol for long periods due to the less crowded and thus, less probability of infection. The fourth category includes rural regions with very low density and thus, the sterilizing process for such regions is carried out over longer periods than other modes.

All locations, of the four categories, are implemented in a database dedicated with the city's locations, in the application server. During the movement of the user, his location is detected using GPS module and is sent to the application server that define the user's region based on the database of the city's locations. Then, the corresponding sterilizing mode is selected and sent to the IoT gateway, to enable the selected sterilizing process. IoT gateway activates the sterilizing mode through the controller connected to the alcohol pump. One more sterilizing mode, besides the four introduced, is deployed for undefined regions and is referred to as the normal sterilizing mode. This mode is activated if the user's location is undefined in the database of the city's locations. The duration and the rate of sterilizing, of each sterilizing mode, are indicated in Tab. 2.

Table 2: Sterilizing modes

\begin{tabular}{llll}
\hline Sterilizing mode & Deployment region & Sterilizing rate & Alcohol rate \\
\hline Mode (1) & Indoor & Every $20 \mathrm{~min}$. & Full \\
Mode (2) & Dense urban & Every $30 \mathrm{~min}$. & Full \\
Mode (3) & Urban & Every $1 \mathrm{hr}$. & Half \\
Mode (4) & Rural & Every $2 \mathrm{hr}$. & Half \\
Mode (5) & Undefined & Every $40 \mathrm{~min}$. & Half \\
\hline
\end{tabular}




\subsection{Experimental Results}

Figs. 4-10 present the real-time and average values of the recorded measures delivered to the application server. Fig. 4 presents the average recorded body temperature, per day, for the first three users; while, Fig. 5 introduces the average recorded body temperature of each user, during the 15 days. The recorded body temperature is compared to the standard human body temperature, i.e., $37^{\circ} \mathrm{C}$, to indicate the health state of the user, and the probability of being infected. Users with daily average body temperature of $37^{\circ} \mathrm{C}$ are marked as healthy, and mentioned in the green database associated with healthy users. While, users with daily average temperature above $37.5^{\circ} \mathrm{C}$ and under $38^{\circ} \mathrm{C}$ are marked as under-risk, and should be checked. Those users are moved to the yellow database dedicated with cases under-observations. Users with body temperature $38^{\circ} \mathrm{C}$, or above, are marked as infected, and moved to the red database developed for high probably infected users that have health issues. Fig. 6 presents the three mentioned databases on the application server with users according to recorded measures, during measuring period. Fig. 7 indicates the average daily measures of $\mathrm{SpO} 2$ level recorded by pulse oximeters of the first three users, for 15 days, and Fig. 8 presents the average measures of SpO2 level of each user, during 15 days. For users with $\mathrm{SpO} 2$ levels higher than 93\%, they are marked as healthy and placed in the green database. Users with $\mathrm{SpO} 2$ level between $90 \%$ and $93 \%$ are moved to the yellow database, and put underobservation, while users with $\mathrm{SpO} 2$ level below $90 \%$ are moved to the red database. As well, Figs. 9 and 10 present the measures of heart rates of each user, per beats, for the testing period.

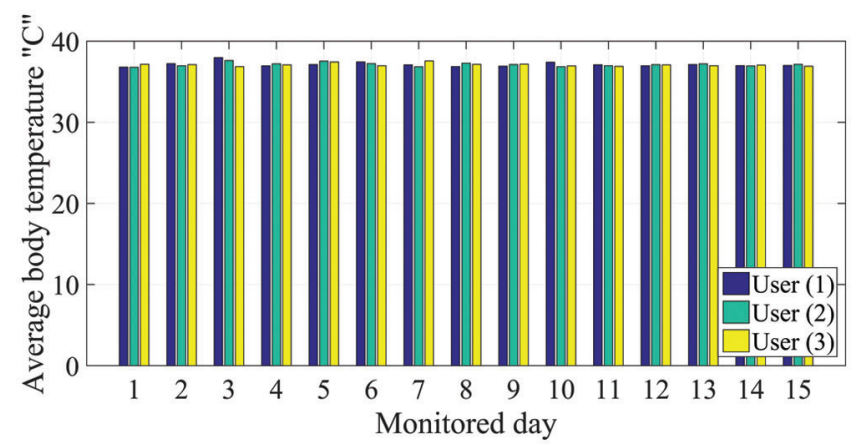

Figure 4: Average body temperature, per day, of the first three users for the measuring period

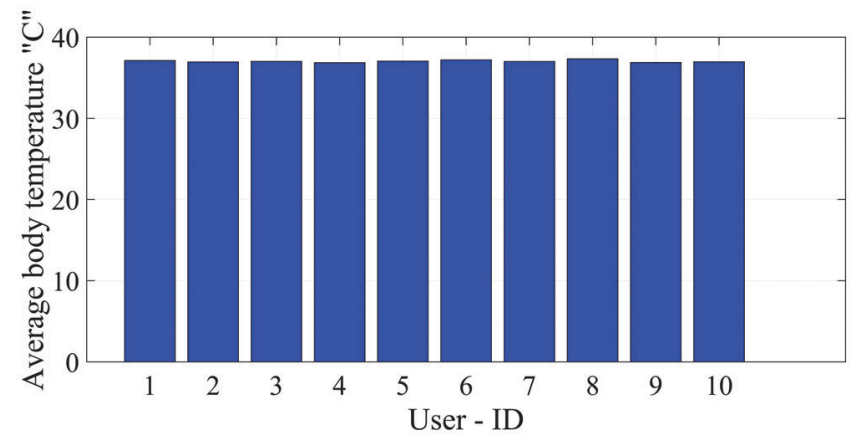

Figure 5: Average body temperature, during the measuring period, of all users 


\begin{tabular}{|c|c|c|c|c|c|c|c|c|}
\hline \multicolumn{3}{|c|}{ Healthy users } & \multicolumn{3}{|c|}{ Under observation } & \multicolumn{3}{|c|}{ Infected users } \\
\hline $\begin{array}{c}\text { User's } \\
\text { II }\end{array}$ & $\begin{array}{c}\text { Reported } \\
\text { Parameter }\end{array}$ & $\begin{array}{c}\text { Last } \\
\text { Modified }\end{array}$ & $\begin{array}{l}\text { User's } \\
\text { II }\end{array}$ & $\begin{array}{c}\text { Reported } \\
\text { Parameter }\end{array}$ & $\begin{array}{c}\text { Last } \\
\text { Modified }\end{array}$ & $\begin{array}{l}\text { User's } \\
\text { II) }\end{array}$ & $\begin{array}{c}\text { Reported } \\
\text { Parameter }\end{array}$ & $\begin{array}{c}\text { Last } \\
\text { Modified }\end{array}$ \\
\hline 22 & All & $\begin{array}{l}9 \mathrm{AAM}- \\
20-04\end{array}$ & 1 & $\overline{\mathrm{T}, \mathrm{O}, \mathrm{HR}}$ & $\begin{array}{l}\text { 9AM-20- } \\
04\end{array}$ & & & \\
\hline 3 & ALL & $\begin{array}{l}9 \mathrm{AM}- \\
20-04\end{array}$ & 5 & $\mathrm{~T}, \mathrm{O}, \mathrm{HR}$ & $\begin{array}{l}9 \mathrm{AM}-20- \\
04\end{array}$ & & & \\
\hline$\overline{44}$ & $\overline{\mathrm{ALL}}$ & $\begin{array}{l}9 \mathrm{AM}- \\
20-04\end{array}$ & $\overline{66}$ & $\overline{T T}$ & $\begin{array}{l}9 \text { AM - 20- } \\
04\end{array}$ & & & \\
\hline 7 & $\mathrm{~T}, \mathrm{O}, \mathrm{HR}$ & $\begin{array}{l}9 \mathrm{AM}- \\
20-04\end{array}$ & 8 & $\mathrm{~T}, \mathrm{HR}$ & $\begin{array}{l}9 \mathrm{AM}-20- \\
04\end{array}$ & & & \\
\hline 9 & $\overline{\mathrm{ALL}}$ & $\begin{array}{l}9 \mathrm{AMM}- \\
20-04\end{array}$ & & & & & & \\
\hline 10 & $\mathrm{~T}, \mathrm{O}, \mathrm{HR}$ & $\begin{array}{l}9 \mathrm{AM}- \\
20-04\end{array}$ & & & & & & \\
\hline
\end{tabular}

Figure 6: Users' databases and records. [T: Temperature measure, O: Oxygen level measure, HR: Heart rate measure]

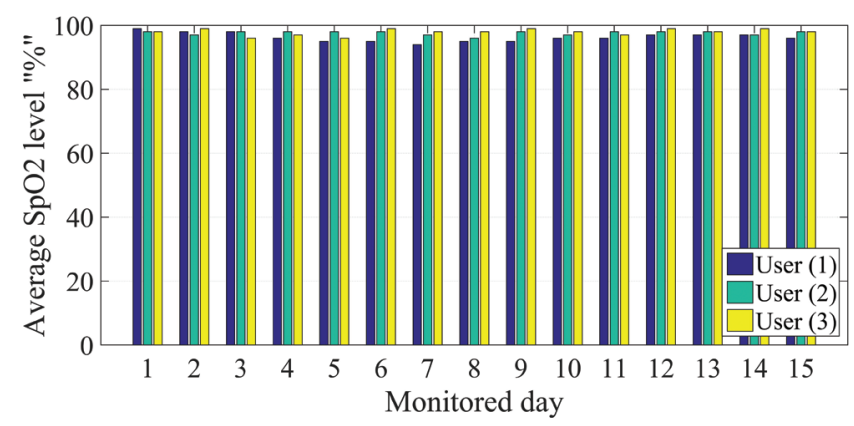

Figure 7: Average $\mathrm{SpO} 2$ level, per day, of the first three users for the measuring period

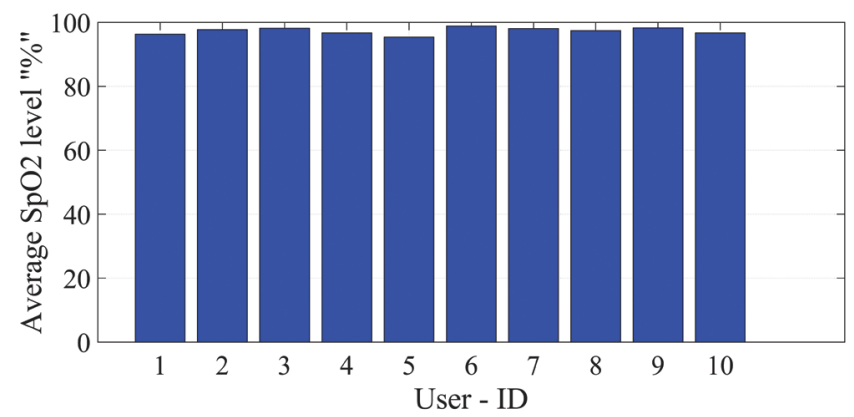

Figure 8: Average $\mathrm{SpO} 2$ level, during the measuring period, of all users

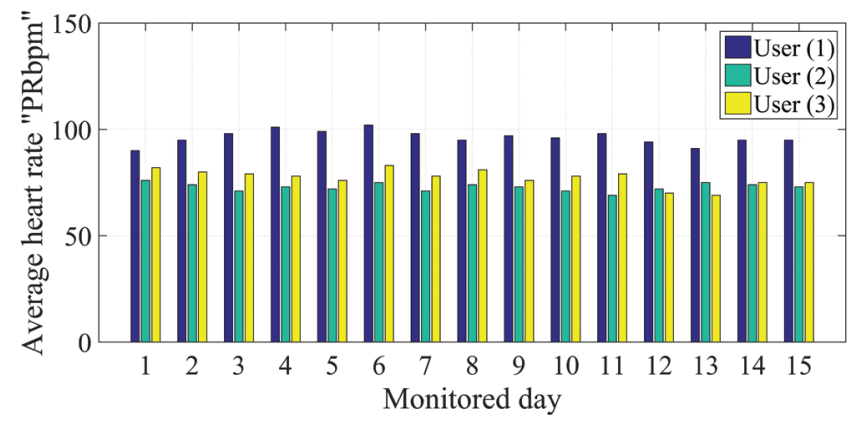

Figure 9: Average heart rate, per day, of the first three users for the measuring period 


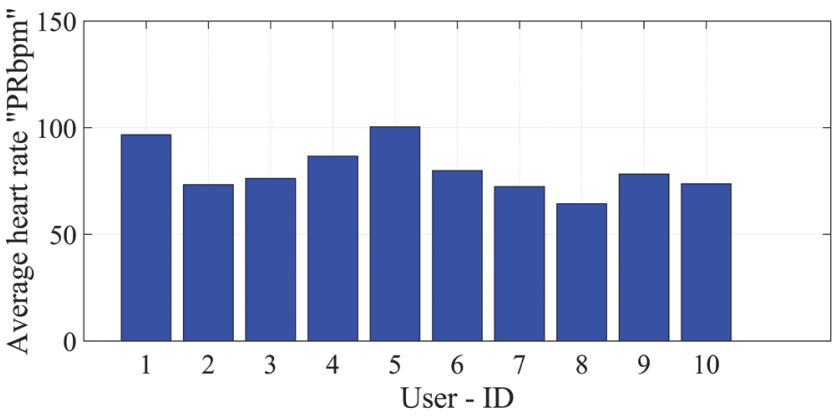

Figure 10: Average heart rate, during the measuring period, of all users

For performance evaluation of the auto-sterilizing system, one hundred locations, belongs to Riyadh city, are selected randomly and sent to the application server to enable the corresponding sterilizing mode. The user is assumed to be stayed in each location for four hours, then moves to the next location. The duration of the sterilizing process is recorded as a measure of the sterilizing mode. Fig. 11 presents the rate of sterilizing process, during four hours for each location.

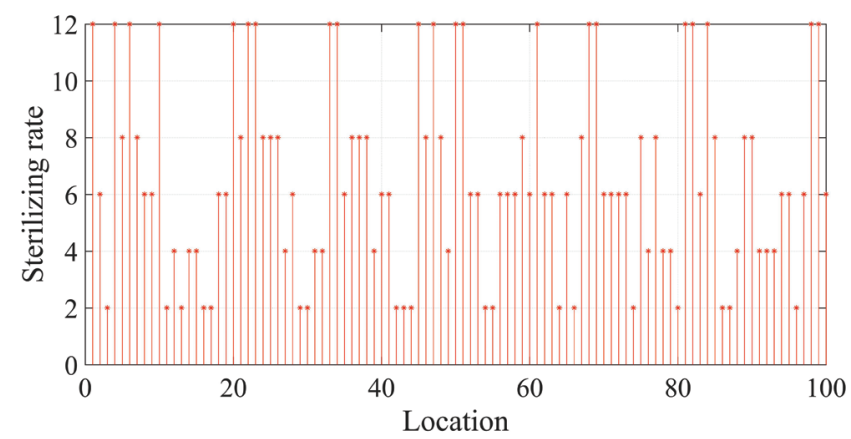

Figure 11: Rate of sterilizing process per each considered location

\section{Discussion}

The proposed IoT-based suit is an efficient way to be used during pandemics, and even as a remote healthcare system in normal days. There are many existing works that consider developing remote healthcare systems and medical systems to monitor COVID-19; however, our proposed work is novel. The novelty of our proposed system comes from many aspects. For better presenting such novelty, and why our proposed system is very efficient, we introduce Tab. 3. Tab. 3 provides a comparison between our proposed suit, other existing works, and medical solutions.

Table 3: Comparison between proposed IoT-based suit and related existing solutions. [No: No evaluation process, E: Experimental evaluation is carried out, S: Simulation process is used for evaluation]

\begin{tabular}{|c|c|c|c|c|c|c|c|c|}
\hline \multirow[t]{2}{*}{ Work } & \multirow{2}{*}{$\begin{array}{l}\text { Remote healthcare } \\
\text { system }\end{array}$} & \multirow{2}{*}{$\begin{array}{l}\text { COVID-19 } \\
\text { Monitoring }\end{array}$} & \multirow[t]{2}{*}{ WBAN } & \multirow{2}{*}{$\begin{array}{l}\text { IoT- } \\
\text { based }\end{array}$} & \multicolumn{3}{|c|}{ Evaluation } & \multirow{2}{*}{$\begin{array}{l}\text { Additional } \\
\text { systems }\end{array}$} \\
\hline & & & & & No & $\mathrm{E}$ & S & \\
\hline [14] & $\mathrm{x}$ & $\sqrt{ }$ & $\mathrm{x}$ & $\sqrt{ }$ & $\mathrm{x}$ & $\sqrt{ }$ & $\mathrm{x}$ & SDN-based \\
\hline [15] & $\mathrm{x}$ & $\sqrt{ }$ & $\mathrm{x}$ & $\sqrt{ }$ & $\sqrt{ }$ & $\mathrm{x}$ & $\mathrm{x}$ & - \\
\hline [17] & $\sqrt{ }$ & $\sqrt{ }$ & $\mathrm{x}$ & $\sqrt{ }$ & $\mathrm{x}$ & $\sqrt{ }$ & $\mathrm{x}$ & - \\
\hline [22] & $\sqrt{ }$ & $\mathrm{x}$ & $\sqrt{ }$ & $\sqrt{ }$ & $\sqrt{ }$ & $\mathrm{x}$ & $\mathrm{x}$ & - \\
\hline
\end{tabular}




\begin{tabular}{|c|c|c|c|c|c|c|c|}
\hline \multirow[t]{2}{*}{ Work } & \multirow{2}{*}{$\begin{array}{l}\text { Remote healthcare } \\
\text { system }\end{array}$} & \multirow{2}{*}{$\begin{array}{l}\text { COVID-19 } \\
\text { Monitoring }\end{array}$} & \multirow[t]{2}{*}{ WBAN } & \multirow{2}{*}{$\begin{array}{l}\text { IoT- } \\
\text { based }\end{array}$} & \multicolumn{2}{|c|}{ Evaluation } & \multirow{2}{*}{$\begin{array}{l}\text { Additional } \\
\text { systems }\end{array}$} \\
\hline & & & & & No & E S & \\
\hline [23] & $\mathrm{x}$ & $\sqrt{ }$ & $\mathrm{x}$ & $\sqrt{ }$ & $\mathrm{x}$ & $x \quad \sqrt{ }$ & - \\
\hline [24] & $\sqrt{ }$ & $\sqrt{ }$ & $\mathrm{x}$ & $\sqrt{ }$ & $\sqrt{ }$ & $x \quad x$ & - \\
\hline [25] & $\mathrm{x}$ & $\sqrt{ }$ & $\mathrm{x}$ & $\sqrt{ }$ & $\mathrm{x}$ & $\sqrt{ } x$ & - \\
\hline [26] & $\mathrm{x}$ & $\sqrt{ }$ & $\mathrm{x}$ & $\sqrt{ }$ & $\sqrt{ }$ & $x \quad x$ & AI-based \\
\hline [27] & $\sqrt{ }$ & $\mathrm{x}$ & $\sqrt{ }$ & $\sqrt{ }$ & & $x \quad x$ & - \\
\hline [28] & $\sqrt{ }$ & $\mathrm{x}$ & $\sqrt{ }$ & $\sqrt{ }$ & $\sqrt{ }$ & $x \quad x$ & - \\
\hline $\begin{array}{l}\text { IoT-based } \\
\text { Suit }\end{array}$ & $\sqrt{ }$ & $\sqrt{ }$ & $\sqrt{ }$ & $\sqrt{ }$ & $\mathrm{x}$ & $\sqrt{ } x$ & $\begin{array}{l}\text { Auto-sterilizing } \\
\text { system }\end{array}$ \\
\hline
\end{tabular}

\section{Conclusions}

Covid-19 spreads, primarily, through the contact with infected persons, when they cough or sneeze, or with an infected surface. A novel way to make a protection against COVID-19 is to stay away, or make yourself isolated from infected people and surfaces. The introduced work provides a novel framework for an auto-sterilizing IoT-based suit that can be used in the era of pandemics. The suit provides the required isolation and sterilizing process to safe health, and work against COVID-19 and other pandemics. The suit deploys a WBAN integrated with IoT system that can be used to monitor healthcare parameters, during daily life and send measures to a remote application server. The proposed healthcare system is efficient, since it is integrated with IoT paradigm, and provides heterogeneous simple interfaces. The developed IoT-based suit thus, provides a reliable cost-efficient healthcare system that can be used in daily life and in the era of pandemics, and moreover, the suit is an auto-sterilized one that can turn the single-use medical suits into reusable ones. The developed IoT-based suit with the integrated WBAN is under prototyping.

Acknowledgement: The authors extend their appreciation to the Deputyship for Research \& Innovation, Ministry of Education in Saudi Arabia for funding this research work through the project number (PNUDRI-Targeted-20-033).

Funding Statement: The authors extend their appreciation to the Deputyship for Research \& Innovation, Ministry of Education in Saudi Arabia for funding this research work through the project number (PNUDRI-Targeted-20-033).

Conflicts of Interest: The authors declare that they have no conflicts of interest to report regarding the present study.

\section{References}

[1] The World health report: Situation report 65, Coronavirus disease (COVID-19), last access on 20-4-2021. [Online]. Available: https://www.who.int/emergencies/diseases/novel-coronavirus-2019/situation-reports.

[2] World Health Organization, Coronavirus disease (COVID-19), last access on 20-04-2021. [Online]. Available: https://www.who.int/emergencies/diseases/novel-coronavirus-2019.

[3] Y. Bai, L. Yao, T. Wei, F. Tian, D. Y. Jin et al., "Presumed asymptomatic carrier transmission of COVID-19," $J A M A$, vol. 323, no. 14, pp. 1406-1407, 2020. 
[4] F. Jiang, L. Deng, L. Zhang, Y. Cai, C. W. Cheung et al., "Review of the clinical characteristics of coronavirus disease 2019 (COVID-19)," Journal of General Internal Medicine, vol. 35, no. 5, pp. 1545-1549, 2020.

[5] F. Aadil, B. Mehmood, N. U. Hasan, S. Lim, S. Ejaz et al., "Remote health monitoring using IoT-based smart wireless body area network," Computers, Materials \& Continua, vol. 68, no. 2, pp. 2499-2513, 2021.

[6] G. Marques, R. Pitarma, N. M. Garcia and N. Pombo, "Internet of things architectures, technologies, applications, challenges, and future directions for enhanced living environments and healthcare systems: A review," Electronics, vol. 8, no. 10, pp. 1081, 2019.

[7] R. P. Singh, M. Javaid, A. Haleem and R. Suman, "Internet of things (IoT) applications to fight against COVID19 pandemic," Diabetes \& Metabolic Syndrome: Clinical Research \& Reviews, vol. 14, no. 4, pp. 521-524, 2020.

[8] K. Kumar, N. Kumar and R. Shah, "Role of IoT to avoid spreading of COVID-19," Int. Journal of Intelligent Networks, vol. 1, no. 5, pp. 32-35, 2020.

[9] M. S. Hajar, M. O. Al-Kadri and H. K. Kalutarage, "A survey on wireless body area networks: Architecture, security challenges and research opportunities," Computers \& Security, vol. 3, no. 6, pp. 10-22, 2021.

[10] Z. Lou, L. Wang, K. Jiang, Z. Wei and G. Shen, "Reviews of wearable healthcare systems: Materials, devices and system integration," Materials Science and Engineering: R: Reports, vol. 140, no. 4, pp. 100523, 2020.

[11] R. Sharma and S. S. Kang, "WBAN for healthcare applications: A survey of current challenges and research opportunities," Journal of Critical Reviews, vol. 7, no. 17, pp. 2444-2453, 2020.

[12] I. Pandey, H. S. Dutta and J. S. Banerjee, "WBAN: A smart approach to next generation e-healthcare system," in Proc. 3rd IEEE Int. Conf. on Computing Methodologies and Communication (ICCMC), Erode, India, pp. 344349, 2019.

[13] Q. Liu, K. G. Mkongwa and C. Zhang, "Performance issues in wireless body area networks for the healthcare application: A survey and future prospects," SN Applied Sciences, vol. 3, no. 2, pp. 1-19, 2021.

[14] Y. Jung and R. Agulto, "A public platform for virtual IoT-based monitoring and tracking of COVID-19," Electronics, vol. 10, no. 1, pp. 1-12, 2021.

[15] Y. Siriwardhana, C. D. Alwis, G. Gür, M. Ylianttila and M. Liyanage, "The fight against the COVID-19 pandemic with 5G technologies," IEEE Engineering Management Review, vol. 48, no. 3, pp. 72-84, 2020.

[16] M. Ndiaye, S. S. Oyewobi, A. M. Abu-Mahfouz, G. P. Hancke, A. M. Kurien et al., "IoT in the wake of COVID19: A survey on contributions, challenges and evolution," IEEE Access, vol. 8, pp. 186821-186839, 2020.

[17] R. Priambodo and T. M. Kadarina, "Monitoring self-isolation patient of COVID-19 with internet of things," in Proc. IEEE Int. Conf. on Communication, Networks and Satellite (Comnetsat), Batam, Indonesia, pp. 87-91, 2020.

[18] World Health Organization, "Requirements and technical specifications of personal protective equipment (PPE) for the novel coronavirus (2019-ncov) in healthcare settings," last access on 23-04-2021. [Online]. Available: https:/www.paho.org/en/documents/requirements-and-technical-specifications-personal-protective-equipmentppe-novel.

[19] Personal Protective Equipment (PPE) Size Charts, last access on 23-04-2021. [Online]. Available: https://www. grainger.com/know-how/safety/ppe-in-the-workplace/ppe-management/kh-personal-protective-equipment-sizecharts-qt-329.

[20] Full Cone Nozzles, last access on 23-04-2021. [Online]. Available: https:/www.pnr.eu/prodotti/full-cone-nozzles/.

[21] CH Full Cone Nozzles, last access on 23-04-2021. [Online]. Available: https:/www.pnr.eu/prodotti/ch/.

[22] Y. Y. Deng, C. L. Chen, W. J. Tsaur, Y. W. Tang and J. H. Chen, "Internet of Things (IoT) based design of a secure and lightweight body area network (BAN) healthcare system," Sensors, vol. 17, no. 12, pp. 2919, 2017.

[23] M. Otoom, N. Otoum, M. A. Alzubaidi, Y. Etoom and R. Banihani, "An IoT-based framework for early identification and monitoring of COVID-19 cases," Biomedical Signal Processing and Control, vol. 62, no. 4, pp. 1-14, 2020.

[24] S. Swayamsiddha and C. Mohanty, "Application of cognitive Internet of Medical Things for COVID-19 pandemic," Diabetes \& Metabolic Syndrome: Clinical Research \& Reviews, vol. 14, no. 5, pp. 911-915, 2020. 
[25] N. Petrović and D. Kocić, "IoT-based system for COVID-19 indoor safety monitoring," IcETRAN, vol. 1, no. 1, pp. 1-6, 2020.

[26] S. Kumar, R. D. Raut and B. E. Narkhede, "A proposed collaborative framework by using artificial intelligenceinternet of things (AI-IoT) in COVID-19 pandemic situation for healthcare workers," Int. Journal of Healthcare Management, vol. 13, no. 4, pp. 337-345, 2020.

[27] A. P. Roy, S. Chatterjee, P. Maji and H. K. Mondal, "Classification of ECG signals for IoT-based smart healthcare applications using WBAN," in Proc. IEEE Int. Sym. on Devices, Circuits and Systems (ISDCS), Howrah, India, pp. 1-4, 2020.

[28] N. Pathak, S. Misra, A. Mukherjee and N. Kumar, "HEDI: Healthcare device interoperability for IoT-based e-health platforms," IEEE Internet of Things Journal, pp. 1, 2021. https://ieeexplore.ieee.org/abstract/ document/9326425. 